\title{
Eficiência técnica da atividade leiteira em Minas Gerais: uma aplicação de regressão quantílica
}

\author{
Ana Carolina Campana Nascimento ${ }^{1}$, João Eustáquio de Lima ${ }^{1}$, Marcelo José Braga ${ }^{1}$, \\ Moysés Nascimento ${ }^{2}$, Adriano Provezano Gomes ${ }^{3}$
}

\footnotetext{
${ }^{1}$ Departamento de Economia Rural da Universidade Federal de Viçosa - Viçosa, Minas Gerais

2 Departamento de Estatística da Universidade Federal de Viçosa - Viçosa, Minas Gerais.

${ }^{3}$ Departamento de Economia da Universidade Federal de Viçosa - Viçosa, Minas Gerais.
}

RESUMO - O objetivo principal neste estudo foi analisar a influência de variáveis técnicas e econômicas sobre os índices de eficiência técnica de produtores de leite de Minas Gerais ao longo de pontos distintos da distribuição dos índices de eficiência utilizando-se a técnica de regressão quantílica. Os índices de eficiência técnica foram estimados com base em um modelo de fronteira estocástica utilizando-se dados de 875 produtores de leite do estado de Minas Gerais coletados no ano de 2005 . Os principais resultados revelaram, na fronteira de produção, que possivelmente está havendo utilização extensiva do fator terra. De modo geral, a variável percentual de vacas em lactação foi a mais relevante na explicação da eficiência técnica em todos os quantis estudados, enquanto o percentual de mão-de-obra familiar utilizado foi importante para explicar apenas os menores níveis de eficiência. Além disso, foi encontrada diferença significativa entre os coeficientes estimados dos quantis em estudo, o que mostra que as variáveis explicativas não têm o mesmo impacto no aumento da eficiência em todos os pontos da distribuição.

Palavras-chave: determinantes da eficiência, fronteira estocástica, pecuária leiteira

\section{Technical efficiency of milk production in Minas Gerais: an application of quantile regression}

\begin{abstract}
The objective of this study was to evaluate the influence of technical and economic variables on the indices of technical efficiency of milk from Minas Gerais throughout distinct points of distribution of the efficiency indices by the technique of quantile regression. The technical efficiency indices were estimated based on a stochastic frontier model, using data from 875 milk producers in Minas Gerais state, Brazil, collected in 2005. The main results from production frontier showed the extensive use of the land factor. Overall, the variable percentage of lactating cows was the more relevant in explaining technical efficiency in all analyzed quantiles, whereas the percentage of household labor was important to explain only the lower levels of efficiency. Moreover, significant differences between the estimated coefficients of the quantiles were found in the study, which showed that the explanatory variables do not have the same impactation on increasing the efficiency at all points of distribution.
\end{abstract}

Key Words: dairy farming, determinants of efficiency, stochastic frontier

\section{Introdução}

A atividade leiteira está entre as seis mais importantes da agropecuária brasileira, desempenhando uma função relevante no desenvolvimento econômico e social do País (Carvalho et al., 2003). O Brasil é o sexto maior produtor de leite do mundo e Minas Gerais, o principal produtor brasileiro (EMBRAPA, 2008). Apesar da expressiva produção, o Brasil não se inclui na lista dos países que produzem leite com elevada produtividade. A baixa produtividade pode ser explicada pela característica da estrutura de produção, em sua maior parte formada por pequenos produtores que utilizam fundamentalmente terra e trabalho.
Transformações no setor agroindustrial brasileiro ocorridas nos anos 90 fizeram crescer a preocupação com a eficiência técnica e econômica do setor, uma vez que a produção tende a se concentrar nos produtores mais eficientes, com maior produtividade e menores custos (Souza, 2003). Desta forma, diversos pesquisadores estudaram a eficiência de produtores de leite, entre eles Ferreira Júnior \& Cunha (2004). Estes autores avaliaram os níveis de eficiência técnica em três sistemas de produção alternativos em Minas Gerais utilizando o modelo de fronteira estocástica de produção. Gonçalves et al. (2008), por outro lado, estimaram a eficiência técnica de produtores de leite de Minas Gerais a partir da técnica de Análise Envoltória de 
Dados. Em ambos os estudos, os determinantes da eficiência técnica foram analisados utilizando-se como base modelos de regressão Tobit.

Em geral, estudos visando identificar os determinantes dos índices de eficiência utilizam modelos de regressão à média, por exemplo os modelos Tobit. Esses modelos, no entanto, desconsideram o fato de que variáveis relacionadas ao sistema de produção podem não influenciar igualmente todos os produtores. Assim, um modelo de regressão único pode não ser adequado, uma vez que, ao estimar apenas um coeficiente para cada variável, considera-se que estas possuem o mesmo efeito no nível de eficiência das unidades produtivas, independentemente de ser alto ou não.

Visando contornar esta limitação, pode-se fazer uso de modelos de regressão quantílica. A estrutura quantílica possibilita, ao estimar diferentes modelos, um para cada quantil de interesse, identificar diferenciações entre os coeficientes, ou seja, diferenças no efeito das variáveis explicativas sobre o nível de eficiência das unidades produtivas. Assim, ao utilizar-se dessa técnica, pressupõe-se que haja a possibilidade de que os fatores determinantes da eficiência de produtores, pouco ou muito eficientes, não sejam os mesmos, ou ainda que estes fatores influenciem de forma diferenciada o nível de eficiência destes produtores.

Desta forma, objetivou-se investigar os determinantes dos índices de eficiência técnica de produtores de leite de Minas Gerais, assistidos pelo Projeto Educampo, com base em modelos de regressão quantílica.

\section{Material e Métodos}

Para o estudo consideram-se dados do tipo cross section, referentes a produtores de leite do estado de Minas Gerais participantes do Projeto de Assistência Técnica EDUCAMPO, que é uma iniciativa do SEBRAE de assistência técnica e gerencial de produtores de leite e outras atividades, que visa melhorias técnicas e ganhos quantitativos e qualitativos ao produto primário, melhorando os indicadores técnicos e econômicos das propriedades.

Foram utilizados dados de 875 produtores de leite de Minas Gerais, entrevistados pelo SEBRAE-MG e FAEMG para a elaboração do Diagnóstico da Pecuária Leiteira do Estado de Minas Gerais em 2005. Esses produtores foram selecionados com base em uma amostragem estratificada segundo os critérios de quantidade produzida em 2004 e o número de produtores em cada estrado de produção (Gomes, 2006).
Para medir a eficiência técnica das unidades produtivas, adotou-se o modelo de fronteira estocástica, proposto por Battese \& Coelli (1992), que apresenta vantagens em relação às alternativas, como as fronteiras determinísticas. Isso se deve ao fato de que as fronteiras determinísticas são baseadas na suposição de que o desvio entre o resultado observado e a fronteira de produção é devido unicamente à ineficiência, ou seja, ignora a possibilidade de que a produção observada pode diferir do potencial por causa de dois outros fatores: choques estocásticos e erros de medição nas variáveis.

A fronteira estocástica de produção proposta pode ser expressa como:

$Y_{i}=f\left(X_{i j} ; \beta\right)+\varepsilon_{i}, \quad i=1,2,3, \ldots, N$ observações

em que $Y_{i}$ é o valor da produção da $i$-ésima propriedade; $f\left(\right.$.) a tecnologia de produção; $X_{i j}$ é a quantidade do $j$-ésimo insumo utilizado pela $i$-ésima propriedade; $\beta$ é o vetor de parâmetros a serem estimados; $\varepsilon_{i}$ representa o erro estocástico, definido como $\varepsilon_{i}=v_{i}-u_{i}$, em que $v_{i}$ e $u_{i}$ são assumidos independentes. Sendo que $v_{i}$ são variáveis aleatórias i.i.d. com distribuição $N\left(0, \sigma_{v}^{2}\right)$ e que representam as variações puramente aleatórias em relação à fronteira, capturando erros de medição, ruídos estatísticos e choques aleatórios fora do controle da propriedade, além dos efeitos das variáveis explicativas não especificadas na função de produção. O termo de erro $u_{i}$, responsável por captar a ineficiência técnica da $i$-ésima propriedade, é assumido como distribuído independentemente com distribuição normal truncada em zero.

Utilizando a parametrização proposta por Battese \& Corra (1977) e Battese \& Coelli (1995), na qual se substitui $\sigma_{v}^{2}$ e $\sigma_{u}^{2}$ por $\sigma^{2}=\sigma_{u}^{2}+\sigma_{v}^{2}$, a ineficiência da propriedade no nível de produto $Y$ é dada por:

$\gamma=\sigma_{u}^{2} /\left(\sigma_{u}^{2}+\sigma_{v}^{2}\right)$

em que: $\sigma^{2}$ é a variância total; $\sigma_{v}^{2}$ é a variância de $v_{i}$; $\sigma_{u}^{2}$ é a variância de $u_{i}$ e $\gamma$ corresponde ao efeito da ineficiência $u_{i}$ na variância do valor da produção, valor que deve estar entre 0 e 1 .

A partir de então, deve-se testar se os efeitos da ineficiência estão ou não presentes no modelo. Para tanto, utilizando o teste da razão de verossimilhança (LR), testa-se a hipótese $\mathrm{H}_{0}: \gamma=0$, ou seja, os efeitos da ineficiência não estão presentes no modelo. Se a hipótese nula não for rejeitada, o termo $u_{i}$ deve ser removido do modelo, deixando uma especificação com parâmetros que podem ser consistentemente estimados por mínimos quadrados ordinários (MQO). 
O valor de $\gamma$ é um indicador da variabilidade relativa entre os desvios da ineficiência e dos ruídos puramente aleatórios, e a contribuição relativa do efeito da ineficiência sobre a variância total é dada por (Coelli et al., 1998):

$$
\gamma^{*}=\frac{\gamma}{\gamma+\left[\frac{(1-\gamma) \pi}{(\pi-2)}\right]}
$$

As fronteiras de produção estocástica podem ser estimadas utilizando-se diferentes formas funcionais, entre elas, as mais utilizadas são as funções Cobb-Douglas e a translogarítmica (translog). Por se tratar de uma das representações funcionais mais simples e largamente utilizadas nas análises de eficiência agrícola (Conceição \& Araújo, 2000; Ferreira Júnior \& Cunha, 2004), utilizou-se, para representar a tecnologia da produção, a função do tipo Cobb-Douglas em sua forma logarítmica. Assim, o modelo de fronteira estocástica (1) a ser estimado pode ser expresso como:

$$
\ln Y_{i}=\ln \beta_{0}+\sum_{j=1}^{4} \beta_{j} \ln X_{i j}+\varepsilon_{i}, \quad i=1,2, \ldots, N \quad \text { e } \quad j=1,2,3,4
$$

em que $Y_{i}=$ produção anual de leite (litros); $X_{1}=$ área utilizada para pecuária (hectares); $X_{2}=$ gasto com mão de obra (R\$); $X_{3}=$ total de vacas (cabeças); e $X_{4}=$ gasto com insumos (reparos de benfeitorias e máquinas; silagem; concentrados; minerais; medicamentos; hormônios; materiais para ordenha; transporte; energia e combustível; inseminação artificial; impostos e taxas) (R\$).

A função de produção foi estimada pelo método da Máxima Verossimilhança e a eficiência técnica, para cada produtor, calculada a partir da seguinte expressão (Coelli, 1996):

$$
E T_{i}=\frac{y_{i}}{\exp \left(x_{i j} \beta\right)}=\frac{\exp \left(x_{i j} \beta_{j}+v_{i}-u_{i}\right)}{\exp \left(x_{i j} \beta_{j}+v_{i}\right)}=\exp \left(-u_{i}\right)
$$

A eficiência técnica assume valores entre zero e um, e indica a distância entre o produto observado da $i$-ésima propriedade $\left(\exp \left(x_{i j} \beta\right)\right)$ e o nível do produto que poderia ser atingindo $\left(y_{i}\right)$ usando a mesma cesta de insumos, caso não houvesse ineficiência.

A estimação dos parâmetros da fronteira estocástica, assim como o cálculo da eficiência das propriedades analisadas, foi realizada utilizando-se o pacote frontier (Coelli, 2008) do software livre $R$ (R Development Core Team, 2009).

Uma vez obtidas as estimativas dos índices de eficiência das unidades produtivas, o passo seguinte consiste em tentar identificar os principais fatores determinantes destes índices. Adicionalmente, busca-se verificar se as unidades produtivas mais eficientes são afetadas diferentemente daquelas de menor nível de eficiência por algumas características em comum. Em outras palavras, deseja-se identificar os impactos de variáveis relacionadas ao desempenho da unidade produtiva ao longo dos diferentes quantis da distribuição dos índices de eficiência e não só a influência média das mesmas. Para tanto, foram estimadas regressões lineares quantílicas em que a variável dependente é o índice de eficiência técnica, obtido por meio da fronteira estocástica de produção (4).

Diferentemente da regressão clássica linear, que estima modelos para funções médias condicionais, o método de Regressão Quantílica (RQ) oferece instrumentos de estimativas de modelos para funções quantis condicionais, o que possibilita a investigação dos impactos das variáveis independentes sobre a eficiência técnica ao longo dos diferentes níveis de eficiência.

A ideia básica é estimar o $p$-ésimo quantil de eficiência condicional sobre as diferentes variáveis explicativas, assumindo que esse quantil pode ser expresso como uma função linear dessas variáveis. O $p$-ésimo quantil de $Y$, a variável dependente, com $0<p<1$, é definido como: $F^{-1}(p)=\inf \{y: F(y) \geq p\}$, em que $F$ é a função de distribuição contínua (não condicional) de $Y: F(y)=\mathrm{P}(Y \leq y)$.

No caso linear, a variável dependente $Y$ é uma função de $X$ da forma: $Y_{i}=X_{i} \beta+\varepsilon_{i}$, em que $\beta$ é o vetor de parâmetros e $\varepsilon_{i}$ é o vetor de erros aleatórios. Assim, a função quantílica condicional da variável dependente $Y$, dada a matriz das variáveis explicativas $X$, pode então ser definida como (Mendes \& Sousa, 2006):

$Q_{p}(y \mid X)=X \beta^{p}, \quad p \in[0,1]$

O estimador de $\beta^{p}$ é encontrado a partir da solução da seguinte função objetivo:

$$
\min _{\beta^{p}} \sum_{y_{i} \geq x_{i} \beta^{p}} p\left|y_{i}-x_{i} \beta^{p}\right|+\sum_{y_{i}<x_{i} \beta^{p}}(1-p)\left|y_{i}-x_{i} \beta^{p}\right|
$$

em que: $\rho$ é a função check definida: $\rho_{p}(z)=\left\{\begin{array}{l}p|z|, \quad z \geq 0 \\ (p-1), z<0\end{array}\right.$

A função objetivo anterior é uma soma ponderada dos desvios absolutos, provendo uma medida local robusta, tal que o vetor de coeficientes estimado não é sensível a observações extremas da variável dependente. Quando os erros não seguem uma distribuição regular, os estimadores de RQ podem ser mais eficientes que os de MQO. As soluções encontradas para os diferentes quantis podem ser interpretadas como variações na variável dependente causadas por mudanças nos regressores em diferentes pontos da distribuição condicional da variável dependente (Buchinsky, 1998, apud Maciel et al., 2003). 
Considerando que as variáveis explicativas não afetam igualmente os níveis de eficiência técnica, foram estimadas regressões para os seguintes quantis: 0,05 (5\%), 0,50 (mediana) e 0,90 (90\%). Sendo o $p$-ésimo quantil condicional do nível de eficiência dado por:

$$
Q_{p}\left(y_{i} \mid z_{1}, z_{2}, z_{3}, z_{4}, z_{5}, z_{6}, z_{7}\right)=\beta_{0}+\sum_{j=1}^{7} \beta_{j} z_{j}, p \in[0,1] \text { e } j=1,2, \ldots, 7 \text {. }
$$

(8)

em que $y_{i}$ = índice de eficiência técnica; $z_{1}=$ tempo em que o produtor está na atividade (anos); $z_{2}=$ escolaridade do produtor (anos de estudo); $z_{3}=$ número de visitas técnicas; $z_{4}=$ percentual de vacas em lactação e $z_{5}=$ área utilizada por vaca (ha/cabeça); $z_{6}=$ percentual de mão-de-obra familiar; e $z_{7}$ margem bruta unitária ( $\mathrm{R} \$$ /litro).

Para verificar se os coeficientes estimados diferem realmente entre as equações estimadas para os três quantis (5, 50 e 95\%), fez-se o uso do teste de Wald, cujas hipóteses a serem testadas são:

$$
H_{0}: \beta_{j}^{\left(p_{i}\right)}=\beta_{j} \forall p_{i} \text { versus } H_{1}: \beta_{j}^{\left(p_{i}\right)} \neq \beta_{j}^{\left(p_{i}^{\prime}\right)} \text {, para } p_{i} \in[0,1] \text { e } j=1,2, \ldots, 7 \text {. }
$$

Sob hipótese nula, a estatística do teste de Wald (W) tem distribuição $\chi^{2}$ com $j$ graus de liberdade (Koenker \& Basset, 1982).

Caso as hipóteses testadas sejam rejeitadas $(\mathrm{P}<0,05)$, tem-se que o impacto das variáveis estudadas nos níveis de eficiência não é o mesmo para todos os produtores analisados, logo, resultados baseados em um modelo de regressão único podem não ser adequados.

A estimação dos parâmetros das regressão para os quantis condicionais e as demais análises foram realizadas utilizando-se o pacote quantreg (Koenker, 2006) do software livre $R$ (R Development Core Team, 2009).

\section{Resultados e Discussão}

De modo geral, percebeu-se grande heterogeneidade entre os produtores amostrados, uma vez que todas as variáveis estudadas possuem grandes valores de coeficiente de variação (CV). Entre estas, as que possuem maiores variabilidade são: custo operacional efetivo (R\$/ano), produção (litros) e gastos com mão-de-obra (R\$ano) (Tabela 1).

Observou-se que, para o modelo de fronteira estimado, apenas o fator terra (área para pecuária) não foi significativo $(\mathrm{P}>0,05)$. Este resultado indica que aumentos da área utilizada para pecuária não contribuirão para aumento na produção de leite (Tabela 2).

Como a função está expressa em termos de logaritmos, seus coeficientes podem ser interpretados diretamente como as elasticidades parciais da produção em relação aos fatores considerados, isto é, como a participação relativa do insumo no produto total (Gujarati, 2006; Chiang \& Wainwright, 2006). De acordo com as elasticidades parciais a variável gasto com insumo foi a que apresentou maior contribuição sobre a elasticidade total da produção, de modo que um acréscimo de $10 \%$ no valor desta variável acarretaria aumento médio da produção de leite de 5,69\%, mantendo os demais fatores constantes, enquanto um aumento de $10 \%$ no total de vacas aumentaria, em média, a produção em 2,26\% (Tabela 2).

Segundo o modelo, a distância entre cada produtor e a fronteira estimada, isto é, o resíduo, pode ser decomposto

\begin{tabular}{|c|c|c|c|c|c|}
\hline Variável & Mínimo & Média & Máximo & Desvio-padrão & CV (\%) \\
\hline Produção anual de leite (L) & $2.380,00$ & $67.266,27$ & $2.927 .086,00$ & $163.031,16$ & 242,37 \\
\hline Área para pecuária (ha) & 0,50 & 56,59 & 1122,00 & 83,02 & 146,70 \\
\hline Custo mão-de-obra (R\$/ano) & 0,00 & $8.666,76$ & $466.680,00$ & $19.139,73$ & 220,84 \\
\hline Total de vacas & 1,00 & 34,12 & $1.000,00$ & 52,14 & 152,80 \\
\hline COE (R\$ano) & 408,30 & $21.138,88$ & $1.336 .694,00$ & $63.357,78$ & 299,72 \\
\hline
\end{tabular}

Tabela 1 - Estatísticas de produção, área para pecuária, número de vacas e gastos com mão-de-obra e insumos, Minas Gerais, 2005

COE = Custo Operacional Efetivo; CV (\%) = coeficiente de variação (\%).

Tabela 2 - Estimativa dos parâmetros da fronteira de produção Cobb-Douglas, Minas Gerais, 2005

\begin{tabular}{lccc}
\hline Variável explicativa & Coeficiente & Erro-padrão & Valor P \\
\hline Intercepto & 2,6772 & 0,1246 & 0,000 \\
Área para pecuária (ha) & $-0,0176$ & 0,0123 & 0,153 \\
Custo mão-de-obra (R\$/ano) & 0,2475 & 0,0187 & 0,000 \\
Total de vacas (cabeças) & 0,2263 & 0,0246 & 0,000 \\
Gasto com insumo (R/ano) & 0,5690 & 0,0149 & 0,000 \\
Sigma quadrado $\left(\sigma^{2}\right)$ & 0,1188 & 0,0145 & 0,000 \\
Gama $(\gamma)$ & 0,5444 & 0,1088 & 0,000 \\
\hline
\end{tabular}


em dois componentes: um componente devido à causas aleatórias e não controladas pelos produtores e, outro devido à ineficiência da unidade produtiva. Os efeitos da ineficiência são captados pelo parâmetro gama $(\gamma)$, cujo valor estimado foi igual a 0,5444 e se mostrou significativo $(\mathrm{P}<0,05)$. Assim, pode-se dizer que existe ineficiência entre os produtores e, ainda, que mais da metade da variação da produção $(54,44 \%)$ se deve à ineficiência técnica dos produtores e não somente a causas aleatórias.

Após a estimação da função de produção estocástica, foram obtidos os índices da eficiência técnica para cada produtor individualmente. Segundo este modelo, as propriedades apresentam índice de eficiência médio de 82,58\% (Tabela 3). A distribuição do índice de eficiência foi definida de forma assimétrica à esquerda (Figura 1). A assimetria da distribuição dos índices de eficiência dá suporte ao estudo com base nos quantis de eficiência e não em relação ao nível médio, dado que a média, neste caso, não constitui a medida que melhor representa a distribuição dos mesmos.
Existe grande variabilidade entre os produtores em relação às variáveis empregadas nos modelos, especialmente quanto à área utilizada por vaca (ha/cabeça), o tempo em que o produtor está na atividade e a escolaridade dos mesmos (Tabela 3). Apesar dessas diferenças, os índices de eficiência técnica possuem pouca dispersão relativa, medida pelo coeficiente de variação $(\mathrm{CV})$, quando comparada às demais variáveis.

Com base nas variáveis apresentadas na Tabela 3, foram estimadas regressões quantílicas para diferentes quantis dos níveis de eficiência, buscando verificar como são influenciados os diferentes produtores em relação às características da administração da propriedade rural. Espera-se que o grau de eficiência técnica esteja relacionado positivamente com as variáveis utilizadas.

Entre os produtores menos eficientes, na regressão estimada para o 5으 quantil (Tabela 4), apenas os coeficientes associados às variáveis tempo que o produtor está na atividade leiteira e número de visitas técnicas não foram significativos $(\mathrm{P}>0,10)$, o que indica que, para aqueles

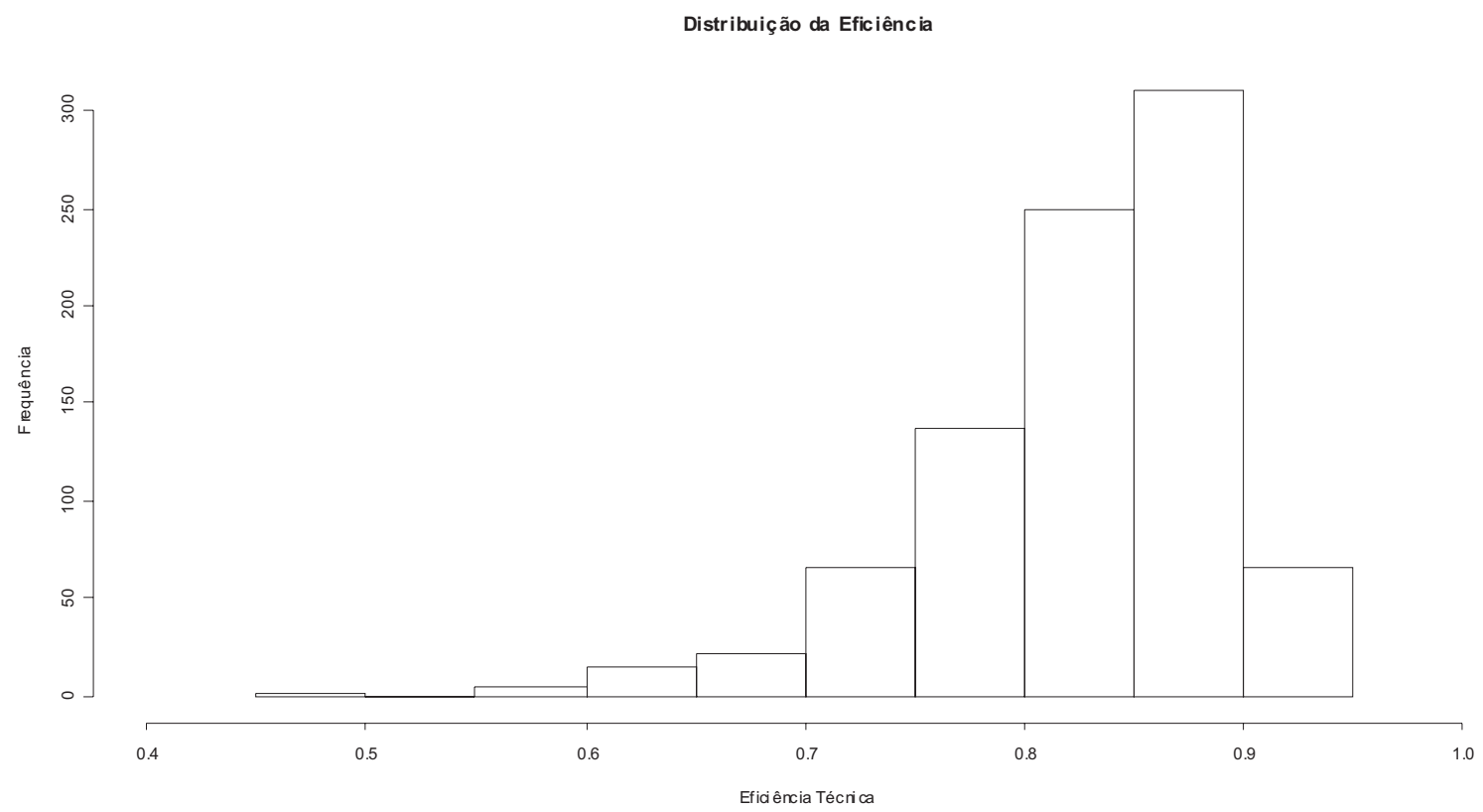

Figura 1 - Distribuição dos índices de eficiência técnica estimados, Minas Gerais, 2005.

Tabela 3 - Estatísticas descritivas das variáveis utilizadas na estimação das regressões quantílicas, Minas Gerais, 2005

\begin{tabular}{|c|c|c|c|c|c|c|}
\hline Variável & Mínimo & Mediana & Média & Máximo & Desvio-padrão & CV (\%) \\
\hline Tempo que o produtor está na atividade (anos) & 1,00 & 18,00 & 19,69 & 68,00 & 14,42 & 73,28 \\
\hline Número de visitas técnicas & 0,00 & 1,00 & 1,88 & 4,00 & 1,10 & 58,59 \\
\hline Percentual de vacas em lactação & 0,30 & 0,67 & 0,66 & 1,00 & 0,11 & 16,75 \\
\hline Área por vaca (ha/cabeça) & 0,02 & 1,33 & 1,80 & 15,90 & 1,60 & 89,19 \\
\hline Percentual de mão de obra familiar & 0,00 & 1,00 & 0,74 & 1,00 & 0,37 & 50,16 \\
\hline
\end{tabular}

CV - coeficiente de variação (\%). 
Tabela 4 - Estimativa dos parâmetros das regressões estimadas, Minas Gerais, 2005

\begin{tabular}{|c|c|c|c|}
\hline \multirow[t]{2}{*}{ Varável explicativa } & \multicolumn{3}{|c|}{ Coeficiente } \\
\hline & 5o quantil & $50^{\circ}$ quantil & $90^{\circ}$ quantil \\
\hline Intercepto & $\begin{array}{l}0,6095^{* * *} \\
(0,0599)\end{array}$ & $\begin{array}{l}0,7367^{* * *} \\
(0,0180)\end{array}$ & $\begin{array}{l}0,8043^{* * *} \\
(0,0154)\end{array}$ \\
\hline Tempo que o produtor está na atividade (anos) & $\begin{array}{l}-0,0006^{\mathrm{ns}} \\
(0,0008)\end{array}$ & $\begin{array}{l}0,00001^{\mathrm{ns}} \\
(0,0002)\end{array}$ & $\begin{array}{c}-0,0003^{*} \\
(0,0002)\end{array}$ \\
\hline Escolaridade do produtor (anos) & $\begin{array}{c}-0,0046^{*} \\
(0,0026)\end{array}$ & $\begin{array}{l}-0,0004^{\mathrm{ns}} \\
(0,0007)\end{array}$ & $\begin{array}{l}0,00002^{\mathrm{ns}} \\
(0,0005)\end{array}$ \\
\hline Número de visitas técnicas & $\begin{array}{l}0,0117^{\mathrm{ns}} \\
(0,0084)\end{array}$ & $\begin{array}{l}-0,0021^{\mathrm{ns}} \\
(0,0029)\end{array}$ & $\begin{array}{l}-0,0022^{\mathrm{ns}} \\
(0,0014)\end{array}$ \\
\hline Percentual de vacas em lactação & $\begin{array}{l}0,1997^{* *} \\
(0,0889)\end{array}$ & $\begin{array}{l}0,1449^{* * *} \\
(0,0192)\end{array}$ & $\begin{array}{l}0,1096^{* * *} \\
(0,0201)\end{array}$ \\
\hline Área por vaca (ha/cabeça) & $\begin{array}{c}-0,0082^{*} \\
(0,0043)\end{array}$ & $\begin{array}{l}-0,0021^{\mathrm{ns}} \\
(0,0023)\end{array}$ & $\begin{array}{l}-0,0003^{\mathrm{ns}} \\
(0,0008)\end{array}$ \\
\hline Percentual de mão-de-obra familiar & $\begin{array}{l}-0,0629^{* * *} \\
(0,0193)\end{array}$ & $\begin{array}{l}-0,0251^{* * *} \\
(0,0068)\end{array}$ & $\begin{array}{l}-0,0090^{\mathrm{ns}} \\
(0,0055)\end{array}$ \\
\hline Margem bruta unitária (R\$/litro) & $\begin{array}{l}0,1239^{* * *} \\
(0,0341)\end{array}$ & $\begin{array}{l}0,1112^{* * *} \\
(0,0237)\end{array}$ & $\begin{array}{l}0,0990^{* * *} \\
(0,0159)\end{array}$ \\
\hline
\end{tabular}

( ) erros-padrão bootstrap das estimativas; ***, ** e * significativos a 0,$01 ; 0,05$; e 0,10 de probabilidade, respectivamente.

produtores menos eficientes, estes não são fatores relevantes para explicar as diferenças observadas nos índices de eficiência. Com relação ao sinal dos coeficientes, aqueles relacionados à escolaridade do produtor e área utilizada por vaca apresentaram sinal negativo, indicando que produtores com maior nível de escolaridade e que utilizam áreas maiores por vaca não estão entre aqueles de maior eficiência neste grupo. Este último resultado pode estar relacionado à utilização excessiva do fator terra. Vale notar que este problema é percebido apenas entre os produtores menos eficientes, uma vez que, para os demais quantis, esta variável não se mostrou significativa para explicar o grau de eficiência dos produtores.

A variável percentual de vacas em lactação foi aquela de maior coeficiente em todos os quantis, indicando que esta é uma variável muito relevante na explicação da eficiência técnica. Todavia, o que se observa é que esta importância diminui conforme os quantis aumentam. Ou seja, quanto menor a eficiência técnica da propriedade, mais determinante é o percentual de vacas em lactação na explicação da mesma. De modo geral, todas as variáveis apresentaram efeito mais intenso sobre o índice de eficiência daquelas propriedades menos eficientes.

Observou-se ainda que o percentual de mão-de-obra familiar utilizado na produção é fator determinante para explicar as diferenças entre os níveis de eficiência técnica, exceto para aqueles produtores mais eficientes (quantil 90). Como resultado, tem-se que aqueles produtores que empregam maior percentual de mão-de obra familiar são também aqueles de menor eficiência técnica. Esta conclusão difere daquela encontrada nos trabalhos de Hallan \& Machado (1994) sobre a atividade leiteira portuguesa e
Dhehibi et al. (2007), que estudaram a ineficiência técnica e suas fontes para o setor cítrico da Tunísia. Nesses estudos, a variável mão-de-obra familiar não foi importante para explicar a eficiência técnica dos produtores. A diferença entre os resultados apresentados evidencia a necessidade de um estudo estratificado com base nos índices de eficiência, uma vez que estimativas baseadas apenas na média da eficiência podem levar à adoção de políticas errôneas.

A variável margem bruta unitária foi também uma variável importante na explicação das diferenças nas eficiências em todos os quantis estimados, enquanto a variável número de visitas técnicas não se mostrou significativa nos três quantis estudados.

Para aqueles produtores mais eficientes (quantil 90), as variáveis mais importantes em diferenciá-los em termos de eficiência foram: tempo que o produtor está na atividade; percentual de vacas em lactação; e margem bruta unitária. Os produtores mais experientes foram neste extrato aqueles de menor eficiência. Novamente, a variável mais importante em diferenciar os produtores e explicar as diferenças entre os níveis de eficiência foi o percentual de vacas em lactação.

Adicionalmente, com base no teste de Wald, observou-se a existência de diferença significativa entre os coeficientes associados à variável percentual de mão-de-obra familiar nos diferentes quantis. Desta forma, tem-se que o impacto desta variável na determinação do índice de eficiência não é o mesmo para todos os produtores estudados, sendo o efeito da mesma maior quanto menor o índice de eficiência. Com relação ao nível de escolaridade do produtor, o teste confirma que somente o quantil 0,05 difere dos demais, sendo que neste grupo de produtores, aqueles com maior nível de escolaridade são também menos eficientes (Tabela 4). 


\section{Conclusões}

Os produtores utilizam o fator terra de forma intensiva, o que indica que a redução na área utilizada para produção de leite poderia ser realizada sem perda de eficiência. O percentual de vacas em lactação mostra-se relevante em termos da explicação da eficiência técnica em todos os quantis. Adicionalmente, o percentual de mão-de-obra familiar mostra-se relevante para se explicar o grau de eficiência apenas de produtores considerados pouco eficientes.

\section{Referências}

BATTESE, G.E.; COELLI, T.J. Frontier production functions, technical efûciency and panel data: with application to paddy farmers in India. Journal of Productivity Analysis, v.3, n.1-2, p.153-169, 1992.

BATTESE, G.E.; CORRA, G.S. Estimation of a Production frontier model: with application to the pastoral zone of Eastern Australia. Australian Journal of Agricultural Economics, v.21, n.3, p.169-179, 1977.

BUCHINSKY, M. Recent advances in quantile regression: a pratical guideline for empirical research. The Journal of Human Resources, v.33, n.1, p.88-126, 1998.

CARVALHO, L.A.; NOVAES, L.P.; MARTINS, C.E et al. [2003]. Importância econômica do leite. Disponível em: <sistemasde producao.cnptia.embrapa.br/fonteshtml/leite/leitecerrado/ importância.html/>. Acesso em: 10 dez. 2008.

CHIANG, A.C.; WAINWRIGHT, K. Matemática para economistas. São Paulo: Campus, 2006. 692p.

COELLI, T.J. A guide to FRONTIER version 4.1: a computer program for stochastic frontier production and cost function estimation. Armidale, Australia: Department of Econometrics, University of New England, 1996. 33p. (CEPA Working Paper, 7).

COELLI, T.J.; HENNINGSEN, A. The frontier package. 2008. Disponível em: http://www.uni-kiel.de/agrarpol/ahenningsen/ frontier/. Acesso em: $28 \mathrm{dez} .2009$.

COELLI, T.J.; RAO, D.S.P.; BATTESE, G.E. An introduction to efficiency and productivity analysis. Norwell: Kluwer Academic Publishers, 1998. 275p.

CONCEIÇÃO, J.C.P.R.; ARAÚJO, P.F.C. Fronteira de produção estocástica e eficiência técnica na agricultura. Revista de Economia e Sociologia Rural, v.38, n.1, p.45-64, 2000.

DHEHIBI, B.; LACHAAL, L.; ELLOUMI, M. et al. Measurement and sources of technical inefficiency in the tunisian citrus growing sector. In: MEDITERRANEAN CONFERENCE OF AGRO-FOOD SOCIAL SCIENTISTS, 1.; EAAE Seminar 'Adding Value to the Agro-Food Supply Chain in the Future Euromediterranean Space', 103., 2007, Barcelona, Espanha. Proceedings... Barcelona, 2007. p.12.

EMPRESA BRASILEIRA DE PESQUISA AGROPECUÁRIA EMBRAPA Gado e Leite. Classificação mundial dos principais países produtores de leite de vaca - 2007. Atualizado em dezembro/2008a. Disponível em: <http:// www.cnpgl.embrapa.br/>. Acesso: 12 jan. 2010.

EMPRESA BRASILEIRA DE PESQUISA AGROPECUÁRIA EMBRAPA Gado e Leite. Ranking da produção anual leite por estado no Brasil - 2007. Atualizado em dezembro/2008b. Disponível em: <http://www.cnpgl.embrapa.br/>. Acesso: 12 jan. 2010.
FARREL, M.J. A measurement of productive efficiency. Journal of the Royal Statistical Society, v.120, n.3, p.254-290, 1957.

FERREIRA JUNIOR, S.F.; CUNHA, N.R.S. Eficiência técnica na atividade leiteira de Minas Gerais: um estudo a partir de três sistemas de produção. Organizações Rurais e Agroindustriais, v.6, n.2, p.46-60, 2004.

GOMES, S.T. Diagnóstico da pecuária leiteira do Estado de Minas Gerais em 2005: relatório de pesquisa. Belo Horizonte: FAEMG, 2006. 156p.

GOMES, S.T. Evolução recente e perspectivas da produção de leite no Brasil. In: GOMES, A.T.; LEITE, J.L.B.; CARNEIRO, A.V. (Eds.). O agronegócio do leite no Brasil. Juiz de Fora: Embrapa, CNPGL, 2001. p.49-61.

GOMES, S.T. Economia da produção de leite. Belo Horizonte: Itambé, 2000. 130p.

GOMES, S.T. Avanços sócio-econômicos em sistemas de produção de leite. In: VILELA, D.; BRESSAN, M.; CUNHA, A.S. (Eds). Restrições técnicas, econômicas e institucionais ao desenvolvimento da cadeia produtiva do leite no Brasil. Brasília: MCT/CNPq/PADCT, 1999. p.139-154.

GONÇALVES, R.M.L.; VIEIRA, W.C.; LIMA, J.E. et al. Analysis of technical efficiency of milk-producing farms in Minas Gerais. Economia Aplicada, v.12, n.2, p.321-335, 2008.

GUJARATI, D. Econometria básica. São Paulo: Campus, 2006 $840 \mathrm{p}$.

HALLAM, D.; MACHADO, F. Efficiency analysis with panel data: A study of Portuguese dairy farms. European Review of Agricultural Economics, v.23, n.1, p.79-93, 1996.

HAO, L.; NAIMAN, D.Q. Quantile regression. Series: Quantitative Applications in the Social Science, 2007. 126p.

KOENKER, R.; BASSETT, G. Regression quantiles. Econometrica, v.46, n.1, p.33-50, 1978.

KOENKER, R.; MACHADO, J. Goodness of fit and related inference processes for quantile regression. Journal of the American Statistical Association, v.94, n.448, p.1296-1310, 1999.

KOENKER, R. Galton, Edgeworth, Frisch, and prospects for quantile regression in econometrics. Journal of Econometrics, n.95, p.347-374, 2000.

KOENKER, R. [2006] Quantile regression in R: A vignette. Disponível em: <http://www.econ.uiuc.edu/ roger/research/rq/ vig.pdf>. Acesso: 12 jun. 2009.

Robust tests for hetroscedasticity based on regression quantiles. Econometrica, v.50, n.1, p.43-61, 1982.

MACIEL, M.C.; CAMPÊLO, A.K.T.N.; RAPOSO, M.C.F. Escolaridade e salário das mulheres no Brasil: uma aplicação de regressão quantílica. Revista Brasileira de Estatística, v.64, n.222, p.7-23, 2003.

MAGALHÃES, K.A.; CAMPOS, R.T. Eficiência técnica e desempenho econômico de produtores de leite no Estado do Ceará, Brasil. Revista de Economia e Sociologia Rural, v.44, n.4, p.695-711, 2006.

MENDES, C.C.; SOUSA, M.C.S. Demand for locally provided public services within the median voter's framework: the case of the Brazilian municipalities. Applied Economics, v.38, n.3, p.239-251, 2006.

R DEVELOPMENT CORE TEAM. R: A language and environment for statistical computing. Vienna, Austria: R Fundation for Statistical Computing, 2009. Disponível em: <http://r-project .org>. Acesso em: 28 dez. 2009.

SOUZA, G.S. Função de produção: uma abordagem estatística com uso de modelos de encapsulamento de dados. Brasília: Embrapa, Informação Tecnológica, 2003. 49p. (Texto para discussão, 17). 